\title{
电力计量自动化
}

伍越

\author{
南方电网广西新电力集团容县公司
}

DOI:10.32629/hwr.v3i12.2609

[ 摘 要] 在科学技术迅猛发展的今天, 电力计量自动化系统逐渐成为电力系统营销自动化建设中不可缺少的一部分。大多数供电公司应该为客 户提供高质量的服务, 并不断提高服务水平。只有这样才能满足未来中国对供电的需求,才能实现灵活互动的业务交流和用电供电, 促进中国供 电企业健康发展。本文针对电力计量自动化系统, 在电力营销中的应用进行深入探讨,明确电力计量自动化系统的实施方案。

[关键词]电力营销; 电力计量自动化; 应用要点

\section{1 电力计量自动化系统的概念}

随着现代化技术的发展, 自动化系统得到了广泛的应用。在电力领域, 自动计量系统系统的普及使用为营销工作带来了技术上的支持, 是电力发 展的必然趋势。电能计量系统的主要分为主站控制系统、通信传输系统和 自动化计量系统, 其中主站控制系统是自动化体系中负责数据收集和处理 部分, 媒介为信道, 可以和各种计量终端连接使用; 通信传输系统即是传输 的媒介, 可以连接主站和计量终端, 具有信息传递的准确性和时效性, 比如 GPRS、CDMA、PSTN等都属于信道的范畴; 而自动化计量系统可以执行核心 控制单元下达的命令, 对检测对象的电能信息进行获取传送, 以达到计量 检测的目的。

\section{2 电力计量自动化系统的组成}

2. 1 大客户大负载管理系统

在电力工业发展的新形势下, 大客户大负荷管理系统为电力公司的 创新和发展提供了可能。通过计算机通信和自动控制系统等一系列高新 技术, 大客户和大负荷管理系统实现对大客户的实时监控。使用大客户 大负荷管理系统, 不但能够实现大功耗供电、大电压, 还可以实时对线路 负载进行监测, 一旦出现问题可以第一时间处理, 保证了用户的安全和 稳定。

\section{2 低压集中抄表系统}

这一系统利用计算机技术和通信技术, 以低压扩频载波为基础, 进行 日常工作。在实际工作过程中, 低压集中抄表系统具有较高的工作效率, 可以及时有效的收集用户电能表的数据信息, 同时将收集的数据进行存 储、处理和分析。由于部分数据由通信介质进行输出, 所以, 该系统的数据 信息不具有实时性,所收集的数据信息为一天之前的信息。低压集中抄表 系统的应用, 在一定程度上减少了人力资源的投入, 降低工作强度。

2. 3 低压集中抄表系统

该系统的主要功能是对集中安装的居民電表表码, 以及相关的电能量 数据进行采集、存储、处理和应用。在供电企业抄表管理过程中实施应用, 该系统可以有效降低人力资源的投入, 对提高电能资源的利用率和远程电 力控制管理, 降低线损率都有重要的意义。

\section{3 电力计量自动化系统在电力营销中的应用}

3. 1 电力计量自动化系统在异常处理与计量装置监测上的应用

电力计量自动化系统可以为所有终端设备提供警报, 并以通讯状况、 采集数据为基础, 利用分析数据为设备提供数据异常、通信异常、数据越 线、数据不全、数据流量大、断相以及失压等警报功能。电力计量自动化 系统的应用, 构建了全方位、实时在线以及覆盖全网的计量远程监测装置 系统, 将电力系统的故障诊断与运行管理模式实现了跨越式发展。将传统 的故障排查工作从 “大海捞针” 式的排查逐渐发展为现在的精确定位和提
前维护, 从根本上提升了工作效率。同时, 改变了计量装置巡检工作的现场 检测模式, 逐渐改变为当前的远程控制智能巡检, 节约大量的人力、物力和 时间, 使报警工作打破传统的人工分析处理模式, 跨越到现在的闭环流程 处理和智能组合处理, 从而实现了对报警岗位的实时监督, 确保报警信息 处理的时效性。

3.2电力计量自动化系统在线损管理方面的应用

在电力公司的管理工作当中, 线损管理占据着不可或缺的重要地位, 特别是线损四分的实时统计工作, 更是过去难以突破的难点。而在电力计 量自动化系统的帮助下, 则诞生了一种前所未有的新型线损管理方案, 在 提升线损管理效率的同时, 还可以有效降低线损的发生率。电力计量自动 化系统在数据完毕之后, 以对象为根据, 全面统计并系统分析配电网的线 损、分压以及分台, 最终自行生成相应的线损统计报表。与此同时, 由主站 系统负责的监测线损异常以及母线电量平衡状况, 能够高效排查并且快速 确定出现线损异常的具体位置及终端。所以不难看出, 在线损管理方面, 电力计量自动化系统的重要性不言而喻。

3. 3 电力计量自动化系统在电负荷特性分析中的应用

电力企业一直为电负荷特性分析所头疼, 传统的手段无法实时有效的 统计和分析数据。而电力计量自动化系统的一个重要组成部分为配变检测 计量系统, 其有效解决了这个问题。一般在公用变用户侧安装本系统, 分为 主站系统和其他部分。通常情况下, 在低压配电变压器、无线局域网技术 中运用配变检测计量系统, 其能够有效统计电能。且配变检测计量系统能 够对输出接口有效控制, 那么如果高低压开关具备了电动执行功能。就可 以有效控制负荷。

3. 4 电力计量自动化系统在服务用户中的运用

负荷预测是在规划电网的过程中一项非常基础的工作, 传统的电力营 销中, 工作人员虽然可以借用电能的计量系统来进行负荷预测, 但是, 其无 法预测到每一位用户用电的负荷数据, 而且预测的结果也不一定准确。但 是, 计量自动化系统却可以对居民用户用电状况进行全面的分析, 进而进 行有效的预测, 为居民用电提供良好的保障。另外, 其还可以有效统计用户 的投诉, 电力公司可以据统计来为用户解决用电问题, 提高服务质量。

3.5 电力计量自动化系统能够起到节能的作用

电力计量自动化系统对用电情况的实时准确监测, 工作人员可以将用 电情况通过网络平台公布于众, 为用户用电提供合理的意见, 帮助用户合 理地用电, 减少不必要资源的浪费。同时, 电力计量自动化系统减少了管理 工作的成本支出。电力计量自动化系统在电力营销工作中的应用, 相对传 统电能计量工作在节能服务工作方面有了明显的优势, 为电力企业管理水 平的提高和经济效益的增加有了很大的帮助。电力计量自动化系统是应运 而生的, 对管理工作的高效开展有着不可估量的作用。 


\title{
浅析水文水资源管理在水利工程中应用
}

\author{
苏琦 \\ 广西正宇工程咨询有限公司 \\ DOI:10.32629/hwr.v3i12.2528
}

[摘 要] 我国虽然水资源丰富,但多数都集中在东南地区,人均水资源遗乏、水资源污染严重,在此情况下,就必须全面加强水文水资源管理工 作。在水利工程中应用水文水资源管理, 可以为水利工程运营管理提供更加精准的信息, 保证水利工程正常运营。在水文水资源管理中, 需要对 水库设计、防洪标准等参数进行对比、优化,提升水利工程抗洪能力、供给能力,这样才能够全面发挥水文水利工程的综合效益。

[关键词] 水文水资源管理; 水利工程; 应用

水利工程在我国社会经济发展具有重要的作用和地位, 在进行水利工 程建设的过程中, 为了提升水利工程建设方案的科学性、合理性与可行性, 确保水利工程的质量, 促进水利工程建设的顺利进行, 必须保证所获取的 相关资料信息的准确性。将水文水资源管理工作运用于水利工程中, 能够 为水利工程项目的开展提供更加准确可靠的资料, 促进水利工程的平稳可 靠运行。

\section{1 水文水资源管理应用于水利工程中重要性}

在水利建设工程中, 重要的基础之一就是水文工作, 其重要组成部分 水文水资源管理又是水利工程建设中的重要依据, 在水利工程建设中起到 了重要的促进作用, 为社会经济效益的提高也发挥着作用。我国因幅员较 广阔, 造成地形、气候和地貎各异, 而且水灾、旱灾也成为最常见的自然灾 害, 对人民群众的生活和工农业生产带来了巨大的影响。对抗灾减灾, 水文 水资源管理起到了重要的非工程性的作用, 成为了水利工程建设的重要建 设依据。水利工程建设受到其管理工作质量水平的影响, 同时也对工农业 生产和人民生活财产安全有着直接的关系。

\section{2 水文水资源管理在水利工程的相关应用 \\ 2. 1 应用内容}

水利工程通常缺少实测流量等资料, 因此水文计算大方向便是以降水 量为依据来对相应地点、时间的洪水线、库容曲线以及水位状态等进行计 算, 从而得到准确的水位信息, 为水文的设计以及校核奠定基础。该项管理 相应结果通常与设计方案联系密切, 水利工程不论是经济还是社会效益均 会遭受影响。通常其计算过程分成如下步骤: 具体的计算流程、针对暴雨、 产流等数值展开计算、对汇流数值进行计算、对调洪数值进行计算。此外

\section{6 电力计量自动化系统在预购电中的应用}

利用遥控功能, 可实现预购电。远程抄表数据直接使用, 然后导入营销 信息数据系统, 对信息数据进行计算, 然后将用户的基本用电信息传输到 网络营业厅, 方便企业全面掌握和分析用电的情况, 进而对电力发电计划 进行合理规划。电力计量自动化系统负载率会由于统计电能的不同, 而每 月定期向那些功率不达标和负载率较低的用户发送用电的提示信息, 通过 这种免费的诊断服务来帮助用户及时的对用电设备进行更换, 以有效达到 节能的目的。

3.7电力计量自动化系统在配变运行监测中的应用

公用变压器在电力供应的过程中能够准确的进行电力数据的监测和 统计, 但是变压器在使用的过程中经常因为电压、功率和负荷之间不能处 于平衡的状态而出现运作的故障。通过利用电力计量自动化系统, 对变压 器运行的检查可以突破线路的距离, 对变压器运行的数据进行及时掌握, 并且根据数据运行的结果对配变运行的状态进行有效的评估, 实现配网生
该过程还涵盖了资料搜集、现场勘查、分析结果以及明确水位等。

2.2 具体应用分析

2.2.1对资料信息进行搜集

对于资料信息搜集工作来说, 其具体任务涵盖如下层面: 首先, 要对水 库诸多方面的资料做到详细把握, 应囊括安全鉴定表、原设计资料、加固 信息等等。其次则应以地形图、相关勘查资料、地理状况等为导向计算出 对应的集水面积、河流特点等。但是很多水利工程多是于山区建立, 原设 计不论是技术还是设备等均相对滞后, 因此多是应用传统形式的地形图, 其实用性并不理想, 若地形图可以紧跟地形变化, 那么便可依靠相应软件 获取准确信息。第三, 即便水库得到加固, 但其后续库容以及面积等变更仍 旧微乎其微。同时若水库得到加固之后, 那么可能受气候等因素使得水库 库容遭受影响, 进而对抗洪能力等造成影响。因此上述问题必须借助现场 勘查等操作才可得到解决。

\subsection{2针对水库现场实施勘查}

以往设计师对水利工程展开设计时并未重视实地勘查环节, 多是依靠 自身猜测等展开设计, 使得部分设计方案很难契合实际需要, 为工程使用 埋下隐患。因此现场勘查对于工程建设来说极为关键。首先, 现场勘查需 要对来水状况加以重视, 明确水库相应的梯度调水、引水渠道等是否契合 水利实际, 了解涵洞应用的闸门控制等。其次, 还应了解水库工程相关文 字、针对其加固情况做到重点分析。最后, 还应针对溢洪道等展开研究, 应包括其材质类别、孔数以及底板情况。

2.2.3对洪水影响进行监测和分析

水利工程需要以地形地貌、实际需要为导向展开设计, 同时要对水库

\section{产运行的效率提升。}

\section{4 结语}

当前我国已经初步进入了信息时代, 所以电力计量自动化系统也应当 大力引进现代先进电子信息技术, 电子信息技术对于电力企业的改革和提 高经济效益都有着重要的意义。所以, 要加强电力计量自动化系统的使用 范围, 并对在使用过程中出现的问题进行及时解决, 有效实现系统优化措 施, 充分发挥电力计量自动化系统的作用。

\section{[参考文献]}

[1]李志新.浅谈电能计量自动化系统在用电检查中的应用 [J].机电信 息,2015(03):3-4.

[2]李良军.计量自动化系统在县供电局电力营销在线稽查中的应用 [J].低碳世界,2017(10):45-46.

[3]李桂萍.浅谈计量自动化系统在电力营销管理中的应用 [J]. 数字技 术与应用,2015(11):76. 